\title{
Modeling Transmission of Tuberculosis with MDR and Undetected Cases
}

\section{Yongqi Liu, ${ }^{1}$ Zhendong Sun, ${ }^{1}$ Guiquan Sun, ${ }^{2}$ Qiu Zhong, ${ }^{3}$ Li Jiang, ${ }^{3}$ Lin Zhou, ${ }^{3}$ Yupeng Qiao, ${ }^{1}$ and Zhongwei Jia ${ }^{4}$}

${ }^{1}$ College of Automation Science and Engineering, South China University of Technology, Guangzhou 510640, China

2 Department of Mathematics, North University of China, Taiyuan 030051, China

${ }^{3}$ Antituberculosis Research Institute of Guangdong Province, Guangzhou 510630, China

${ }^{4}$ National Institute on Drug Dependence, Peking University, Beijing 100083, China

Correspondence should be addressed to Zhongwei Jia, urchinjj@163.com and Zhendong Sun, zdsun@scut.edu.cn

Received 22 February 2011; Accepted 10 April 2011

Academic Editor: Guang Zhang

Copyright (c) 2011 Yongqi Liu et al. This is an open access article distributed under the Creative Commons Attribution License, which permits unrestricted use, distribution, and reproduction in any medium, provided the original work is properly cited.

This paper presents a novel mathematical model with multidrug-resistant (MDR) and undetected TB cases. The theoretical analysis indicates that the disease-free equilibrium is globally asymptotically stable if $R_{0}<1$; otherwise, the system may exist a locally asymptotically stable endemic equilibrium. The model is also used to simulate and predict TB epidemic in Guangdong. The results imply that our model is in agreement with actual data and the undetected rate plays vital role in the TB trend. Our model also implies that TB cannot be eradicated from population if it continues to implement current TB control strategies.

\section{Introduction}

China is among the highest TB burden countries in the world and is second only to India. The number of MDR-TB cases is about one third of that of the world [1, 2]. For an MDRTB patient, the cost for the treatment is usually 10-100 times higher than that of a common TB patient [3]. Moreover, undetected TB patients pose threat to others, which plays a major role in mycobacterium transmission among the general population [4]. It is no doubt that the earlier the diagnosis and treatment, the better in attenuating the transmissions. Clearly, the understanding of the mechanism and contribution of undetected patients in transmission is important to conduct targeted interventions and control TB decease.

Blower et al. proposed a transmission dynamics among the cases infected by drugsensitive and the drug-resistant strains, respectively [5]. Many subsequent investigations 
focused on similar topics with various perspectives about the role of drug-resistant strain $[3,6-8]$. However, these works failed to independently identify the role of the undetected patients in TB transmission. motivated by the previous work, we carefully divide the infectious cases into undetected cases and timely detected cases in this paper. This improved classification enables us to interpret the real situation in a better manner. To analyze the stability of the disease-free equilibrium in our transmission model, we introduce a novel Lyapunov function. We prove that the disease-free equilibrium is globally asymptotically stable when the basic reproduction number is less than one, and a forward (transcritical) bifurcation exists otherwise.

While Guangdong is a developed province of China, the number of TB cases in Guangdong is about $10 \%$ of that of national TB cases. Mass migrants, MDR, and HIV/AIDS coinfections make the situation of TB transmission very complex in this region. We apply our model to describe the TB situation in Guangdong and simulate the distribution and predication. The simulations indicate that undetected cases do play an important role in TB transmission.

The remainder of the paper is organized as follows. The proposed TB model is formulated in Section 2. Stability criteria of the equilibria are presented in Section 3. Data analysis and simulation studies are provided in Section 4 . Finally, Section 5 concludes with discussions and conclusions.

\section{Model Description}

In this paper, we consider the transmission diagram shown in Figure 1 and the mathematical model is given by

$$
\begin{gathered}
\frac{d S}{d t}=A-\beta_{1} I_{U} S-\beta_{2} I_{G} S-\beta_{3} I_{R} S-\mu S \\
\frac{d E}{d t}=\beta_{1} I_{U} S+\beta_{2} I_{G} S+\beta_{3} I_{R} S-\left(\mu+k+k_{1}\right) E+\omega R, \\
\frac{d I_{U}}{d t}=k E-\left(\mu+\mu_{D}+v\right) I_{U}, \\
\frac{d I_{G}}{d t}=v m I_{U}+k_{1} p E-\left(\mu+\mu_{D}^{\prime}+r_{1}\right) I_{G}-\sigma I_{G}, \\
\frac{d I_{R}}{d t}=v(1-m) I_{U}+k_{1}(1-p) E-\left(\mu+\mu_{D}^{\prime \prime}+r_{2}\right) I_{R}+\sigma I_{G}, \\
\frac{d R}{d t}=r_{1} I_{G}+r_{2} I_{R}-(\mu+\omega) R,
\end{gathered}
$$

where $S, E, I_{U}, I_{G}, I_{R}$, and $R$ represent the numbers of cases of susceptible, latent, undetected infectious TB, detected drug-sensitive infectious TB, detected infectious MDR-TB, and the treated, respectively. $A$ is the recruitment rate. $\beta_{1}, \beta_{2}$, and $\beta_{3}$ are the transmission rates of the undetected infectious, detected infectious drug-sensitive, and detected infectious MDR-TB cases for susceptible individuals, respectively. $\mu$ is the per capita natural mortality rate. $\mu_{D}$, $\mu_{D}^{\prime}$, and $\mu_{D}^{\prime \prime}$ are the death rates (associated to TB) of undetected infectious, detected infectious drug-sensitive, and detected infectious MDR-TB cases, respectively. $\left(k+k_{1}\right)$ is progressive rate from latent to infectious, with $k_{1}$ being the detected rate from latent cases to undetected 


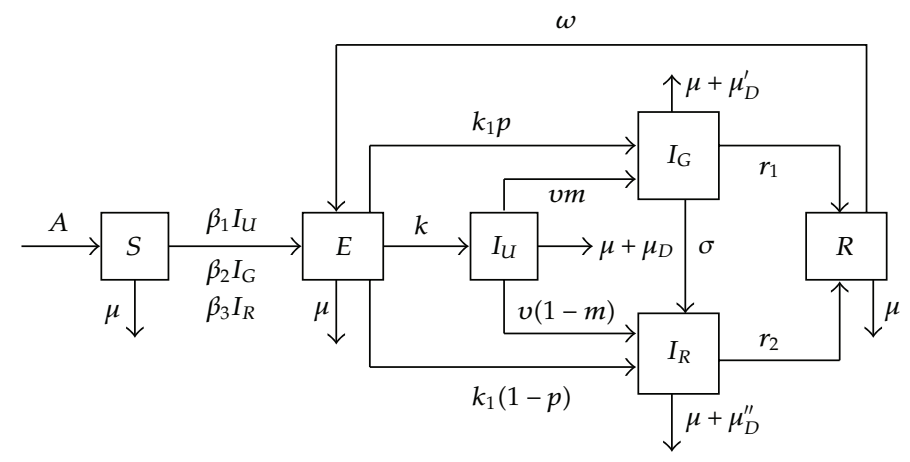

Figure 1: Transfer diagram of the model.

cases in two weeks (from onset of symptoms to treatment, see [2]). The infectivity of the infected patient is strongest in the first two weeks after being infected. $v$ is the per capita detected rate from undetected to detected, called the delayed detected rate. $r_{1}, r_{2}$ are the treatment rates of detected infectious drug-sensitive and detected infectious MDR-TB cases, respectively. $\omega$ is the reactivation rate from treated to latent. $\sigma$ is the treatment failure rate of detected drug-sensitive infectious patients who have been incompletely treated. We assume that patients without incomplete treatment will develop into MDR-TB as in [5] and the infectious transmission rate is bilinear.

Let $N(t)=S+E+I_{U}+I_{G}+I_{R}+R$ be the total population. It follows from the system equations that

$$
\frac{d N(t)}{d t} \leq A-\mu N
$$

which implies that

$$
\varlimsup_{t \rightarrow \infty} N(t) \leq \frac{A}{\mu}
$$

Therefore, we have an attractor of system (2.1); that is,

$$
P=\left\{\left(S, E, I_{U}, I_{G}, I_{R}, R\right) \in R_{+}^{6}: S+E+I_{U}+I_{G}+I_{R}+R \leq \frac{A}{\mu}\right\}
$$

It is clear that $P$ is an invariant set with respect to system (2.1) and any solution of system (2.1) initiated elsewhere will finally converge to the set.

\section{Stability Analysis}

\subsection{The Basic Reproductive Number}

In many epidemiological models, there exists a threshold condition, which determines whether an infection will be eliminated or become endemic. The basic reproduction number 
represents the average number of secondary infections caused by a single infected individual in susceptible population. For simplicity, denote

$$
\begin{gathered}
a=\mu+\mu_{D}+v, \quad b=\mu+\mu_{D}^{\prime}+r_{1}, \quad c=\mu+\mu_{D}^{\prime \prime}+r_{2}, \\
a^{\prime}=k m v+k_{1} p a, \quad b^{\prime}=k m v \sigma+k_{1}(1-p) a(b+\sigma)+k_{1} p a \sigma+k v(1-m)(b+\sigma),
\end{gathered}
$$

and further

$$
\begin{gathered}
X=A(\mu+\omega)\left[\beta_{1} k c(b+\sigma)+\beta_{2} c a^{\prime}+\beta_{3} b^{\prime}\right], \\
Y=\mu \omega\left(r_{1} c a^{\prime}+r_{2} b^{\prime}\right) \\
Z=\mu(\mu+\omega) a(b+\sigma) c\left(\mu+k_{1}+k\right) .
\end{gathered}
$$

Note that

$$
Z-Y=\mu(\mu+\omega) a(b+\sigma) c\left(\mu+k_{1}+k\right)-\mu \omega\left(r_{1} c a^{\prime}+r_{2} b^{\prime}\right)>0
$$

Computing the basic reproduction number yields

$$
\begin{aligned}
R_{0} & =\frac{X+Y}{Z} \\
& =\frac{A(\mu+\omega)\left[\beta_{1} k c(b+\sigma)+\beta_{2} c a^{\prime}+\beta_{3} b^{\prime}\right]+\mu \omega\left(r_{1} c a^{\prime}+r_{2} b^{\prime}\right)}{\mu(\mu+\omega) a c(b+\sigma)\left(\mu+k_{1}+k\right)} .
\end{aligned}
$$

Obviously, $R_{0}$ is increasing with respect to $\beta_{1}, \beta_{2}, \beta_{3}$, and $\omega$. Note also that $\beta_{2}, \beta_{3}$ are less than $\beta_{1}$ after treatment. As a result, for sakes of deceasing $R_{0}$, it is important to increase the detected rate for disease control.

Evidently, system (2.1) has a disease-free equilibrium $P_{1}(A / \mu, 0,0,0,0,0)$. When $R_{0}>$ 1 , it has yet a unique endemic equilibrium $P_{2}\left(S^{*}, E^{*}, I_{U^{\prime}}^{*} I_{G^{\prime}}^{*} I_{R^{\prime}}^{*} R^{*}\right) \in P^{0}$, the interior of $P$. Routine calculations show that

$$
I_{U}^{*}=\frac{\mu A(\mu+\omega) k c(b+\sigma)(X+Y-Z)}{X(Z-Y)}=\frac{\mu k c A(\mu+\omega)(b+\sigma)\left(R_{0}-1\right)}{X Z(Z-Y)}
$$

and $S^{*}, E^{*}, I_{G^{\prime}}^{*} I_{R^{\prime}}^{*}$ and $R^{*}$ could be obtained accordingly.

\subsection{Proofs of Main Theorems}

Theorem 3.1. The disease-free equilibrium $P_{1}$ of system (2.1) is globally asymptotically stable if $R_{0} \leq$ 1 and is unstable if $R_{0}>1$. 
Proof. In the region $P$, we take the Lyapunov function

$$
L\left(S, E, I_{U}, I_{G}, I_{R}, R\right)=S-S_{0}-S_{0} \ln \frac{S}{S_{0}}+E+\frac{\omega}{\omega+\mu} R+\frac{h}{\mu(\mu+\omega) c} I_{R}+h_{1} I_{G}+h_{2} I_{U},
$$

where

$$
\begin{gathered}
S_{0}=\frac{A}{\mu} \\
h=A \beta_{3}(\omega+\mu)+\mu \omega r_{2} \\
h_{1}=\frac{A \beta_{2}(\mu+\omega) c+\sigma h+\mu \omega c r_{1}}{\mu(\mu+\omega) c(b+\sigma)}, \\
h_{2}=\frac{A \beta_{1}(\mu+\omega)(b+\sigma) c+h(b+\sigma) v(1-m)}{\mu(\mu+\omega) a(b+\sigma) c}+\frac{\mu \omega c r_{1} v m+h \sigma v m+A \beta_{2}(\mu+\omega) c v m}{\mu(\mu+\omega) c(b+\sigma)} .
\end{gathered}
$$

It can be verified that $L\left(S, E, I_{U}, I_{G}, I_{R}, R\right) \geq 0$ and $L\left(S, E, I_{U}, I_{G}, I_{R}, R\right)=0$ if and only if $\left(S, E, I_{U}, I_{G}, I_{R}, R\right)=(A / \mu, 0,0,0,0,0)=P_{1}$. This means that the function is positive definite.

Routine calculation shows that

$$
\frac{d L}{d t}=\left(2-\frac{S_{0}}{S}-\frac{S}{S_{0}}\right) A+\frac{\mu+k_{1}+k}{Z}(X+Y-Z) E .
$$

When $R_{0} \leq 1$, we have

$$
\frac{d L}{d t}=A\left(2-\frac{S_{0}}{S}-\frac{S}{S_{0}}\right)+\frac{\mu+k_{1}+k}{Z}(X+Y-Z) E \leq 0
$$

It is clear that $d L / d t=0$ if and only if either $S=S_{0}, E=0$ when $R_{0}<1$ or $S=S_{0}$ when $R_{0}=1$. The maximum invariant set in the region $\left\{\left(S, E, I_{U}, I_{G}, I_{R}, R\right) \in P, d L / d t=0\right\}$ is $\left\{P_{1}\right\}$ when $R_{0} \leq 1$. It follows from the LaSalle invariant set theory that $P_{1}(A / \mu, 0,0,0,0,0)$ is globally asymptotically stable when $R_{0} \leq 1$.

The Jacobian matrix of system $(2.1)$ at $P_{1}(A / \mu, 0,0,0,0,0)$ is computed to be

$$
J\left(P_{1}\right)=\left(\begin{array}{ll}
A_{1} & A_{2} \\
A_{3} & A_{4}
\end{array}\right)
$$


where

$$
\begin{gathered}
A_{1}=\left(\begin{array}{ccc}
-\mu & 0 & -\beta_{1} \frac{A}{\mu} \\
0 & -\left(\mu+k+k_{1}\right) & \beta_{1} \frac{A}{\mu} \\
0 & k & -a
\end{array}\right), \\
A_{2}=\left(\begin{array}{ccc}
-\beta_{2} \frac{A}{\mu} & -\beta_{3} \frac{A}{\mu} & 0 \\
\beta_{2} \frac{A}{\mu} & \beta_{3} \frac{A}{\mu} & w \\
0 & 0 & 0
\end{array}\right) \\
A_{3}=\left(\begin{array}{ccc}
0 & k_{1} p & m v \\
0 & k_{1}(1-p) & v(1-m) \\
0 & 0 & 0
\end{array}\right) \\
A_{4}=\left(\begin{array}{ccc}
-(b+\sigma) & 0 & 0 \\
\sigma & -c & 0 \\
r_{1} & r_{2} & -(\omega+\mu)
\end{array}\right) .
\end{gathered}
$$

It can be obtained that $\left|J\left(P_{1}\right)\right|=Z-(X+Y)$. When $R_{0}>1$, we have $\left|J\left(P_{1}\right)\right|<0$. Then, it can be seen that $J\left(P_{1}\right)$ has at least an eigenvalue with positive real part, thus $P_{1}$ is unstable if $R_{0}>1$. By the Lyapunov method, equilibrium $P_{1}$ is unstable.

Remark 3.2. When $R_{0}>1$, we see that $J\left(P_{1}\right)$ has at least one eigenvalue with positive real part and at least one eigenvalue with negative real part, hence $P_{1}$ is a saddle point with a stable manifold and an unstable manifold. Solutions starting sufficiently close to $P_{1}$ must leave a neighborhood of $P_{1}$, except those on invariant $T$-axis which approach $P_{1}$ along the $T$-axis.

Theorem 3.3. System (2.1) has a forward bifurcation at $\beta_{1}=\beta_{1}^{*}$, and the endemic equilibrium $P_{2}$ is locally asymptotically stable if $0<R_{0}-1 \ll 1$.

Proof. We proceed along the lines as Theorem 4.1 and Remark 1 in [6]. Suppose that $\beta_{1}$ is chosen to be a bifurcation parameter. By solving $R_{0}=1$ with variable $\beta_{1}$, we obtain

$$
\beta_{1}^{*}=\frac{\mu a c(\mu+\omega)(b+\sigma)\left(\mu+k_{1}+k\right)}{A k c(\mu+\omega)(b+\sigma)}-\frac{A(\mu+\omega)\left(\beta_{2} c a^{\prime}+\beta_{3} b^{\prime}\right)+\mu \omega\left(r_{1} c a^{\prime}+r_{2} b^{\prime}\right)}{A k c(\mu+\omega)(b+\sigma)} .
$$

When $\beta_{1}=\beta_{1}^{*}$, we have $\left|J\left(P_{1}\right)\right|=0$. When $\beta_{1}>\beta_{1}^{*}$, we have $\left|J\left(P_{1}\right)\right|<0$. When $\beta_{1}<\beta_{1}^{*}$, we have $\left|J\left(P_{1}\right)\right|>0$. Denote $J\left(P_{1}, \beta_{1}^{*}\right)=\left.J\left(P_{1}\right)\right|_{\beta_{1}=\beta_{1}^{*}}$.

First, we will show that 0 is a single eigenvalue of $J\left(P_{1}, \beta_{1}^{*}\right)$. To this end, let $F(\lambda)=$ $\operatorname{det}\left(J\left(P_{1}, \beta_{1}^{*}\right)-\lambda I\right)$ and $J\left(P_{1}, \beta_{1}^{*}\right)-\lambda I=\left(a_{i j}(\lambda)\right), i, j=1, \ldots, 6$. Denote the derivative of $F(\lambda)$ 
by $F^{\prime}(\lambda)$ and the algebraic complement of $a_{i i}(\lambda)$ by $A_{i i}(\lambda)$ for $i, j=1, \ldots, 6$. Simple calculation gives

$$
F^{\prime}(0)=-\left[A_{11}(0)+A_{22}(0)+A_{33}(0)\right]-\left[A_{44}(0)+A_{55}(0)+A_{66}(0)\right]>0 .
$$

As a result, 0 is simple eigenvalue of $J\left(P_{1}, \beta_{1}^{*}\right)$. It can be seen that all the other eigenvalues of $J\left(P_{1}, \beta_{1}^{*}\right)$ have negative real parts.

Denote, respectively, the right and the left eigenvectors of $J\left(P_{1}\right)$ corresponding to the zero eigenvalue by

$$
L=\left(L_{1}, L_{2}, L_{3}, L_{4}, L_{5}, L_{6}\right)^{T}, \quad M=\left(M_{1}, M_{2}, M_{3}, M_{4}, M_{5}, M_{6}\right)
$$

It follows from

$$
J\left(P_{1}, \beta_{1}^{*}\right) L=0, \quad M J\left(P_{1}, \beta_{1}^{*}\right)=0,
$$

that

$$
\begin{gathered}
L_{1}=\frac{Y-Z}{\mu^{2} k c(\omega+\mu)(b+\sigma)} L_{3}, \quad L_{2}=\frac{a}{k} L_{3}, \quad L_{3} \neq 0, \\
L_{4}=\frac{a^{\prime}}{k(b+\sigma)} L_{3}, \quad L_{5}=\frac{b^{\prime}}{k c(b+\sigma)} L_{3}, \quad L_{6}=\frac{c r_{1} a^{\prime}+r_{2} b^{\prime}}{k(\omega+\mu) c(b+\sigma)} L_{3}, \\
M_{1}=0, \quad M_{2} \neq 0, \\
M_{3}=\frac{A \beta_{1}^{*} c(\mu+\omega)(b+\sigma)+m v h_{3}}{\mu a c(\mu+\omega)(b+\sigma)} M_{2}+\frac{(1-m) v(b+\sigma)\left(A \beta_{3}(\omega+\mu)+\mu \omega r_{2}\right)}{\mu a c(\mu+\omega)(b+\sigma)} M_{2}, \\
M_{4}=\frac{h_{3}}{\mu c(\mu+\omega)(b+\sigma)} M_{2}, \quad M_{5}=\frac{A \beta_{3}(\omega+\mu)+\mu \omega r_{2}}{\mu c(\mu+\omega)} M_{2}, \quad M_{6}=\frac{\omega}{\omega+\mu} M_{2},
\end{gathered}
$$

where

$$
h_{3}=A c \beta_{2}(\omega+\mu)+A \beta_{3} \sigma(\omega+\mu)+\mu \omega r_{2} \sigma+\mu \omega c r_{1} .
$$

Assume that $M_{2}>0$ and $L_{3}>0$ which are always possible up to a sign change of $L$ and $M$. The conditions in [6] are satisfied. Furthermore, routine calculation shows that the quantities as in [6] are

$$
\begin{aligned}
& M_{2} L_{1}\left(\beta_{1}^{\prime} L_{3}+\beta_{2} L_{4}+\beta_{3} L_{5}\right)<0, \\
& M_{2} \frac{A}{\mu}\left(\beta_{1}^{\prime} L_{3}+\beta_{2} L_{4}+\beta_{3} L_{5}\right)>0,
\end{aligned}
$$


Table 1: Model parameters for simulations.

\begin{tabular}{|c|c|c|}
\hline Parameters & Estimate & Source \\
\hline$A=$ recruitment rate & $1.05 * 10^{6}$ & GHSY \\
\hline $\begin{array}{l}\beta_{1}=\text { infection rate by the undetected } \\
\text { infectious }\end{array}$ & $2.2 * 10^{-7}$ & \\
\hline $\begin{array}{l}\beta_{2}=\text { infection rate by the detected } \\
\text { infectious drug sensitive }\end{array}$ & $2 * 10^{-8}$ & \\
\hline $\begin{array}{l}\beta_{3}=\text { infection rate by the detected } \\
\text { infectious MDR-TB }\end{array}$ & $6 * 10^{-8}$ & \\
\hline$\mu=$ natural mortality rate & 0.015 & GHSY \\
\hline$k=$ endogenous reactivation rate & $2 * 10^{-3}$ & ARIG \\
\hline$k_{1}=$ detected rate in time & $3.2 * 10^{-3}$ & ARIG \\
\hline$\omega=$ reinfected rate from the treated & $6 * 10^{-2}$ & \\
\hline $\begin{array}{l}v=\text { detected rate from the undetected } \\
\text { infectious }\end{array}$ & 0.6 & ARIG \\
\hline$\mu_{D}=$ death rate associated to $\mathrm{TB}$ & 0.25 & \\
\hline$\mu_{D}^{\prime}=$ death rate associated to $\mathrm{TB}$ & 0.0063 & ARIG \\
\hline$\mu_{D}^{\prime \prime}=$ death rate associated to $\mathrm{TB}$ & 0.028 & \\
\hline $\begin{array}{l}r_{1}=\text { treatment rate of detected } \\
\text { infectious drug sensitive }\end{array}$ & 0.94 & ARIG \\
\hline $\begin{array}{l}r_{2}=\text { treatment rate of detected } \\
\text { infectious MDR-TB }\end{array}$ & 0.6 & ARIG \\
\hline $\begin{array}{l}\sigma=\text { treatment failure rate of detected } \\
\text { drug sensitive }\end{array}$ & 0.04 & ARIG \\
\hline $\begin{array}{l}m=\text { proportion of detected infectious } \\
\text { drug sensitive in time }\end{array}$ & 0.916 & ARIG \\
\hline $\begin{array}{l}p=\text { proportion of detected infectious } \\
\text { drug sensitive with delay }\end{array}$ & 0.916 & ARIG \\
\hline
\end{tabular}

respectively. By applying [6], we show that system (2.1) has a forward bifurcation at $\beta_{1}=\beta_{1}^{*}$. Therefore, when $\beta_{1}>\beta_{1}^{*}$ (i.e., $R_{0}>1$ ) with $\beta_{1}$ close to $\beta_{1}^{*}, P_{2}$ is locally asymptotically stable.

\section{Simulations}

We use TB data provided by the Antituberculosis Research Institute of Guangdong Province (ARIG). We collect TB data from 1992 to 2008 and estimate the trend of TB cases in the near future in Guangdong province. The World Bank Loaned China Infectious and Endemic Disease Control Project has been carried out since 2000. According to the project plan, Guangdong province would implement the DOTS with the coverage rate $100 \%$, the discovery rate $80 \%$, and the treatment rate $90 \%$ in 2010. In this section, we carry out some simulations to describe the dynamic behavior of system (2.1), and predict the tendency of the TB disease. Setting one year as unit time, first we determine the values of the parameters using the data from 1992 to 2002, and then, we verify the model using the data from 2003 to 2008.

Assume that $\beta_{1}=0.00000022, \beta_{2}=0.00000002, \beta_{3}=0.00000006$, which are adjusted from the literature $[2,5]$. Furthermore, assume that $k+k_{1}=0.0052$ based on the estimation that $10 \%$ of the latent cases will develop into infectious person in 20 years [9]. The detected rate in time $k_{1}$ and the treatment failure rate $\sigma$ that we estimate are, respectively, $3.2 \times 10^{-3}$ and 0.04 . Among the detected infectious patients, about $8.32 \%$ are MDR-TB patients from our data, so 


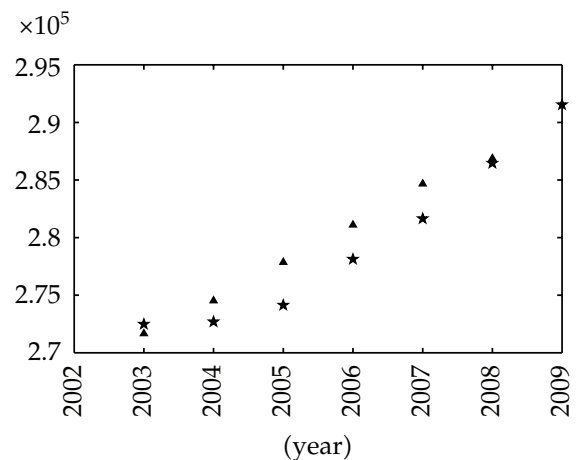

$\star$ Predicted TB infection cases

- Actual TB infection cases

(a)

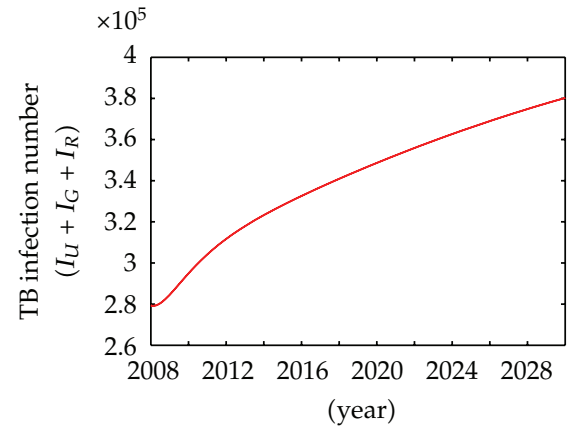

(b)

Figure 2: Trend simulation and prediction of the infection cases in Guangdong, China. (a) Contrast diagram of predicted TB infection cases and actual TB infection cases. (b) Prediction result of TB infection cases.

we assume that $m=p=0.916$. The other parameters in Table 1 can be estimated or derived from our data using the weighted average method. In Table 1, GHSY means Guangdong health statistical yearbook. sizes

Using the investigated data in 2002 in Guangdong province as the initial population

$$
\begin{aligned}
& {\left[S(02), E(02), I_{U}(02), I_{G}(02), I_{R}(02), R(02)\right]} \\
& \quad=[41241970,33600080,158990,105250,5290,46810],
\end{aligned}
$$

we predict the TB situation from 2003 to 2008 in Figure 2(a). $\star$ and $\boldsymbol{\Delta}$ represent predicted infection TB cases and actual TB cases, respectively. That the prediction basically coincides with the actual infection number demonstrates the ability of our model in describing the TB disease.

To obtain simulation results in a more accurate manner, we adjust some parameters by using data from 1992 to 2008 . Let $A=950000, \mu=0.013, \mu_{D}^{\prime}=0.0023$, and $\mu_{D}^{\prime \prime}=0.02$ in the following simulations. Using the other parameter values given in Table 1 and the data of 2008

$$
\begin{aligned}
& {\left[S(08), E(08), I_{U}(08), I_{G}(08), I_{R}(08), R(08)\right]} \\
& \quad=[40241000,38600000,98990,155200,25200,96800],
\end{aligned}
$$

as the initiate state, we obtain the predicted data shown in Figures 2(b) and 3. We observe that the population of the undetected infected individuals slowly tends to a stable endemic equilibrium. The total TB infected number will be $3.28 \times 10^{5}$ in 2015 . This indicates a very severe situation, which concurs with the warning from the leading experts [10]. 

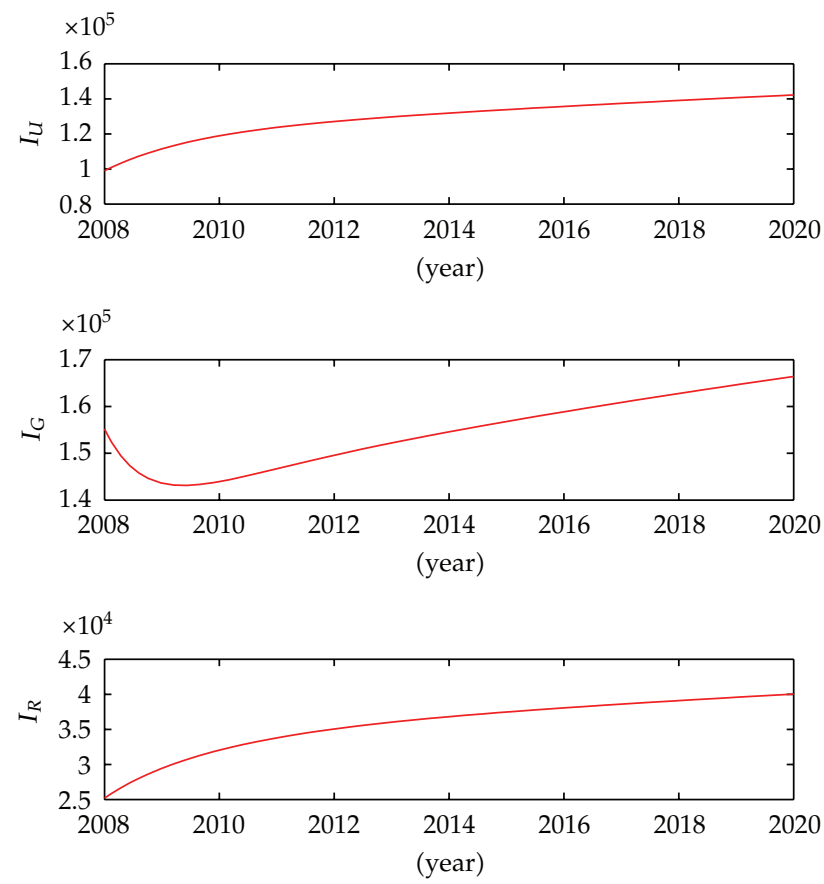

Figure 3: Simulation of system (2.1) in Guangdong province, China. Disease persistence in the population. Using the parameters in Table 1 , we obtain $R_{0}=3.5846>1$.

Figure 4(a) is a graphical representation of total TB infection number under three different delay discovery rates. It is clear that only when $v \geq 0.9$, the infection number will keep relatively stable.

Figure $4(\mathrm{~b})$ is a graphical representation of total TB infection number with different $k_{1}$ 's. It shows the influence of the detected rate in time. If the delay rate $k /\left(k+k_{1}\right)$ is about $80 \%$, the total infection number rapidly raises. On the contrary, if the detected rate in time $k_{1}=0.0042$, the amount of the infection number will basically remains stable from 2014. It can be seen that the detected rate in time is an important factor for disease control.

Figure 4(c) is a graphical representation of total TB infection number with different treatment rate of the MDR-TB patients. If $r_{2}=0.8$, the total infection number will be smaller than $3 \times 10^{5}$ in 2012 .

\section{Discussions and Conclusions}

In this work, combining stability analysis with bifurcation identification, we have investigated the dynamical behavior of a TB model and taken into account two features, the undetected TB cases and MDR-TB cases. The model had an influence up on TB prevention and control. We found that there are two possible steady states: the healthy and the infected equilibria. We proved the global asymptotic stability of the disease-free equilibrium when the basic reproduction number is equal to or less than unity. The local asymptotic stability of the endemic equilibrium has been also established when the basic reproduction number is bigger than unity. A more delicate analysis based on the center manifold theory indicated that a forward bifurcation existed under a proper condition. Forward bifurcation showed 


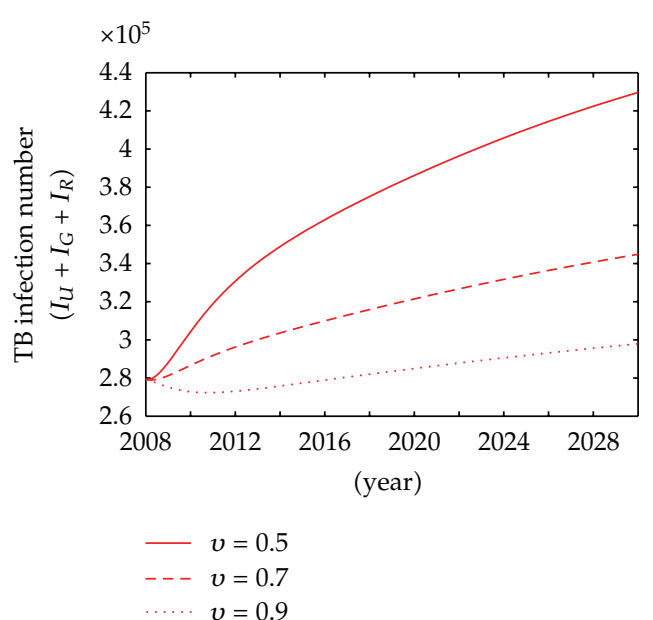

(a)

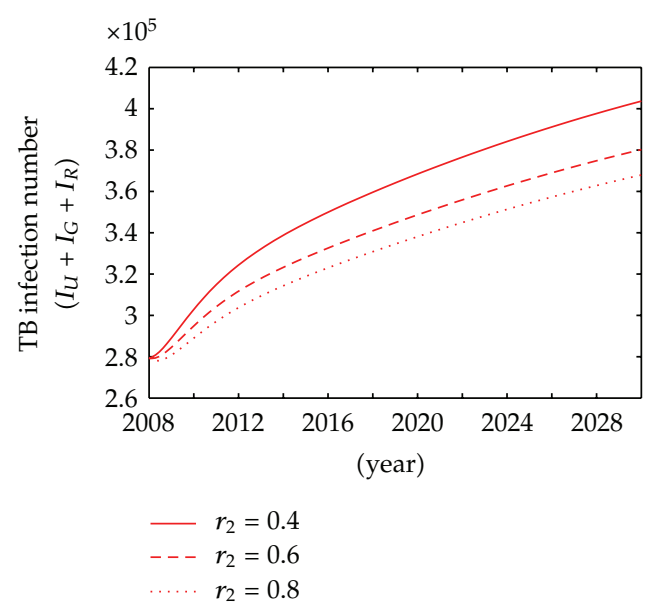

(c)

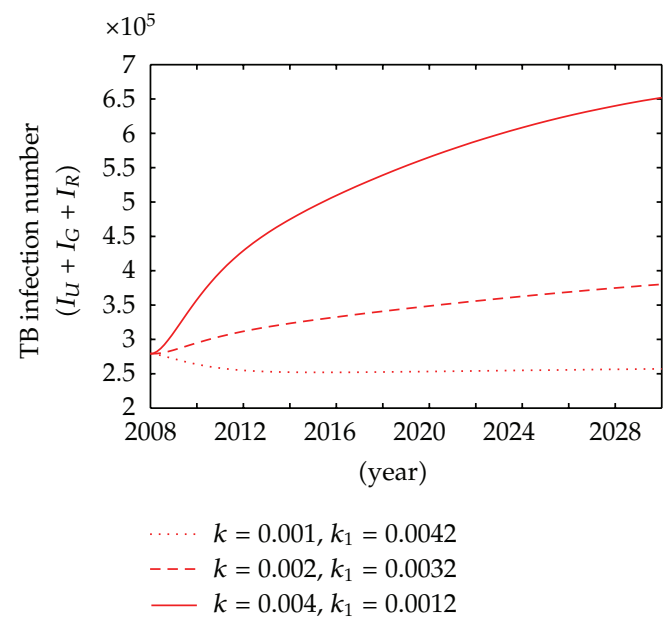

(b)

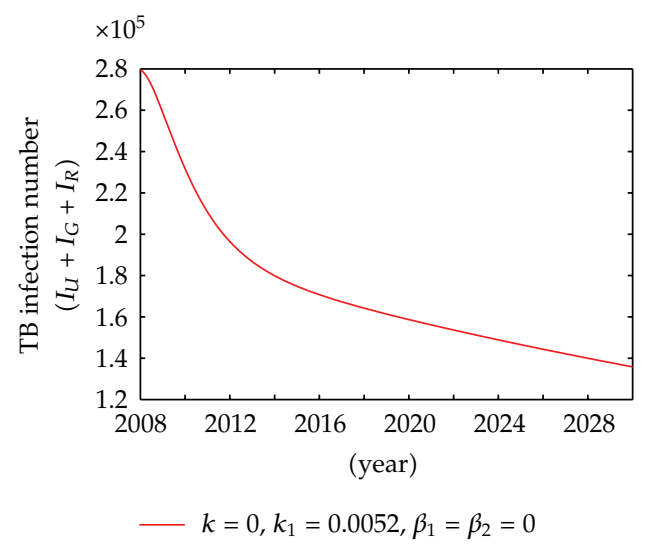

(d)

Figure 4: Simulations with different parameters. (a) Different discovery rates. (b) Different detected rates in time. (c) Different treatment rates of the MDR-TB patients. (d) All TB infection cases are detected in time.

that there existed a stable endemic equilibrium when the disease-free equilibrium become unstable. Furthermore, this work does not give proofs of global asymptotical stability of the endemic equilibrium.

The number of the MDR-TB cases in China is about one third as that of the world. As a province with the one-tenth TB cases in China, Guangdong province suffers much from the TB disease. From Figure 3, we can find the undetected infected patients slowly increase. A primary reason for this is that the detection ratio has been improved thanks to the implementation of the World Bank Loaned China Infectious and Endemic Disease Control Project. However, due to the facts that the total population of residence is huge in Guangdong (about 100 millions) and more than 20 percent of residents are temporary immigrants from the rural inland, the number of infected people will slowly go up. The huge population of rural migrants makes the in-time detection more difficult. We have analyzed the data 
collected by ARIG and carried out simulations based on our model. First, the parameters of the model were identified, which justifies the important role that undetected cases play in the model. Second, we predicted the trend of number of TB infection cases in 20 years and found that it was difficult to eliminate the TB disease under current conditions. Third, we analyzed the possibility of controlling the disease by improving the detected rates and other factors. In particular, when every patient can be detected in time, that is, $k=0, k_{1}=0.0052$, and the detected patients have no infectiousness, then we can obtain $R_{0}=0.1879<1$ even if $v=0.6$ and $r_{2}=0.6$. Under these assumptions, the solutions of the system will tend to the disease-free equilibrium $P_{1}$, and the TB disease will finally die out as indicated in Figure $4(\mathrm{~d})$. This once again shows the importance of the detection rate in TB control.

\section{Acknowledgments}

The authors are grateful to the referees for the valuable suggestions and numerous comments which improve the quality of the paper. This work was supported by China's National Science and Technology Special Projects during the 11th Five-Year Plan Grant no. 2008ZX10003-007 and the National Natural Science Foundation of China under Grants nos. 60925013 and 30973981.

\section{References}

[1] WHO, "Global tuberculosis control: surveillance, planning, financing," 2008.

[2] Z. Jia, X. Li, W. Cao, S. Chen, and C. Dye, "Temporal-spatial model and risk factor evaluation for Tuberculosis," Tech. Rep., Beijing University of Posts and Telecommucation, 2008.

[3] C. Dye and B. G. Williams, "Criteria for the control of drug-resistant tuberculosis," Proceedings of the National Academy of Sciences of the United States of America, vol. 97, no. 14, pp. 8180-8185, 2000.

[4] M. W. Borgdorff, "New measurable indicator for tuberculosis case detection," Emerging Infectious Diseases, vol. 10, no. 9, pp. 1523-1528, 2004.

[5] S. M. Blower, P. M. Small, and P. C. Hopewell, "Control strategies for tuberculosis epidemics: new models for old problems," Science, vol. 273, no. 5274, pp. 497-500, 1996.

[6] C. Castillo-Chavez and B. Song, "Dynamical models of tuberculosis and their applications," Mathematical Biosciences and Engineering, vol. 1, no. 2, pp. 361-404, 2004.

[7] C. Castillo-Chavez and Z. Feng, "To treat or not to treat: the case of tuberculosis," Journal of Mathematical Biology, vol. 35, no. 6, pp. 629-656, 1997.

[8] A. B. Gumel and B. Song, "Existence of multiple-stable equilibria for a multi-drug-resistant model of Mycobacterium tuberculosis," Mathematical Biosciences and Engineering, vol. 5, no. 3, pp. 437-455, 2008.

[9] T. C. Porco and S. M. Blower, "Quantifying the intrinsic transmission dynamics of tuberculosis," Theoretical Population Biology, vol. 54, no. 2, pp. 117-132, 1998.

[10] N. S. Zhong, "Half Chinese population will be with tubercle bacillus," People's Daily, March 2010 (Chinese). 


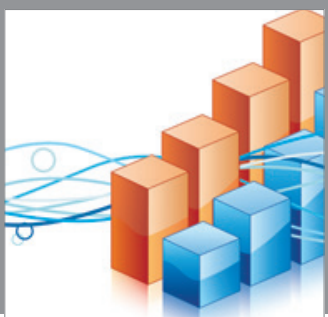

Advances in

Operations Research

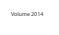

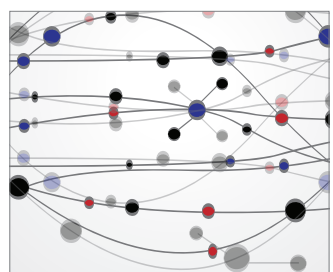

\section{The Scientific} World Journal
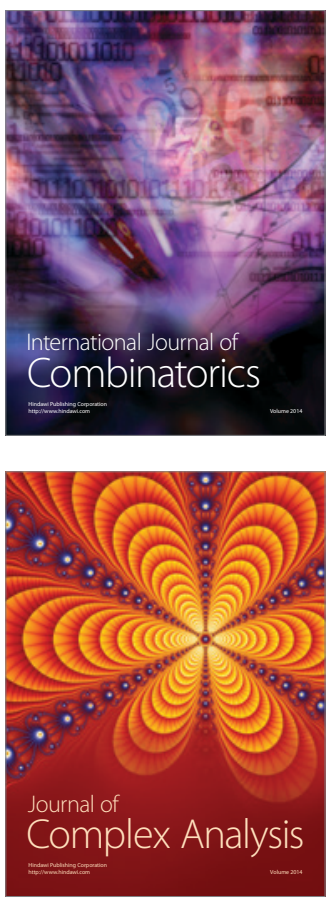

International Journal of

Mathematics and

Mathematical

Sciences
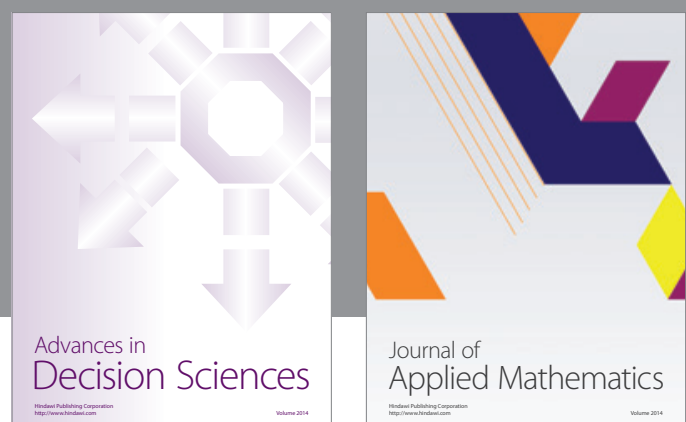

Journal of

Applied Mathematics
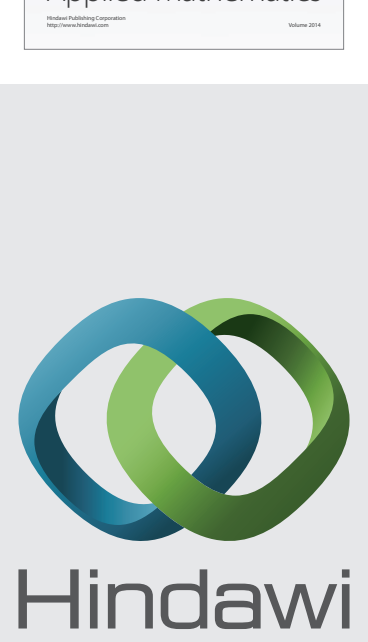

Submit your manuscripts at http://www.hindawi.com
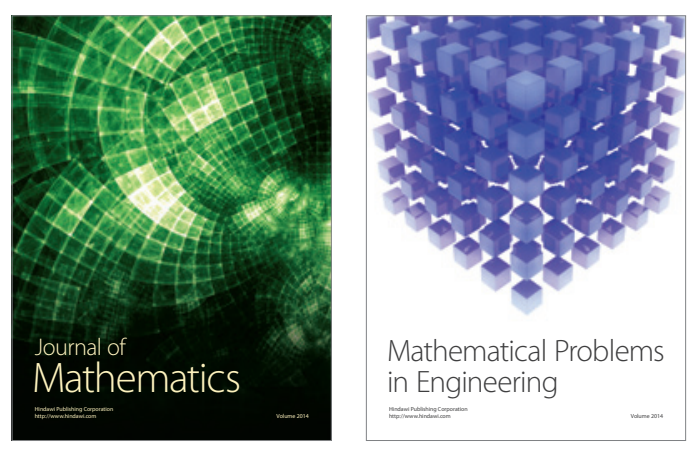

Mathematical Problems in Engineering
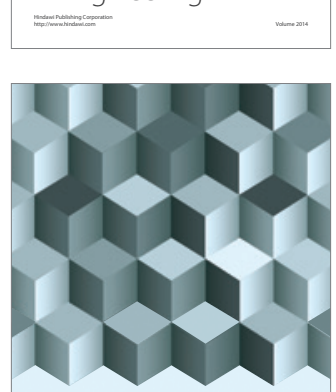

Journal of

Function Spaces
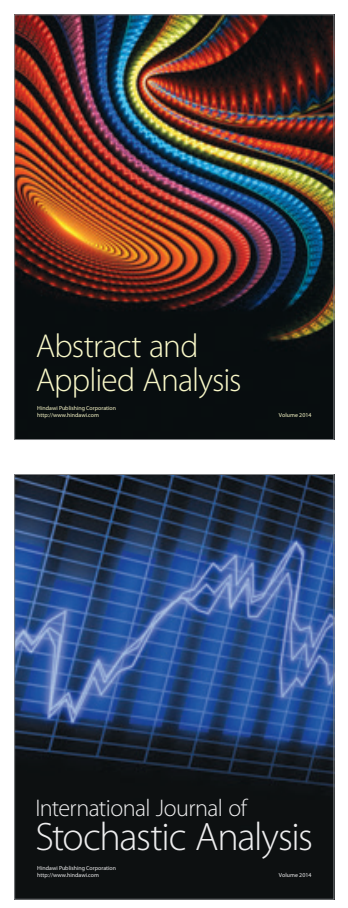

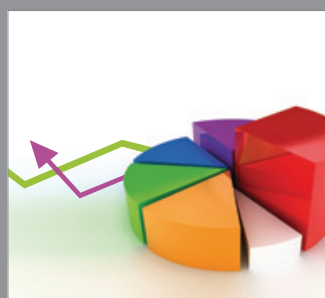

ournal of

Probability and Statistics

Promensencen
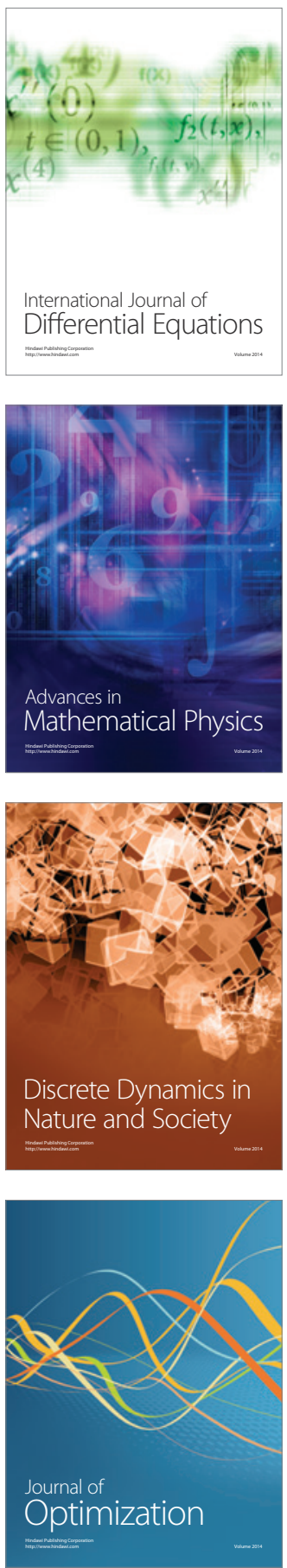